\title{
Editorial
}

\section{Advances in Antenna Design and System Technologies for Next Generation Cellular Systems}

\author{
Chih-Peng Li, ${ }^{1}$ Sumei Sun, ${ }^{2}$ Ding-Bing Lin, ${ }^{3}$ Jung-Chieh Chen, ${ }^{4}$ and Qiang Chen ${ }^{5}$ \\ ${ }^{1}$ Department of Electrical Engineering and Institute of Communications Engineering, National Sun Yat-sen University, \\ 70 Lien-Hai Road, Kaohsiung 80424, Taiwan \\ ${ }^{2}$ Modulation and Coding Department, Institute for Infocomm Research, 1 Fusionopolis Way, \\ No. 21-01 Connexis South Tower, Singapore 138632 \\ ${ }^{3}$ Department of Electronic Engineering and Graduate Institute of Computer and Communication Engineering, \\ National Taipei University of Technology, No. 1, Section 3, Chuang-Hsiao E. Road, Taipei 10608, Taiwan \\ ${ }^{4}$ Department of Optoelectronics Communication Engineering, National Kaohsiung Normal University, No.116, \\ Heping 1st Road, Kaohsiung 80201, Taiwan \\ ${ }^{5}$ Department of Communications Engineering, Graduate School of Engineering, Tohoku University, 6-6-05, \\ Aramaki Aza Aoba, Aoba-ku, Sendai, Miyagi 980-8579, Japan
}

Correspondence should be addressed to Chih-Peng Li; cpli@faculty.nsysu.edu.tw

Received 12 December 2012; Accepted 12 December 2012

Copyright (C) 2013 Chih-Peng Li et al. This is an open access article distributed under the Creative Commons Attribution License, which permits unrestricted use, distribution, and reproduction in any medium, provided the original work is properly cited.

With the increasing demand for ubiquitous multimedia services, next-generation cellular systems are expected to provide higher data rates and robustness to the changing wireless environment. As a fundamental part of any cellular system, an antenna plays a primary role and its performance is directly related to the quality of communication signal. However, the overall system performance is determined by the total performance of each component, such as antenna and baseband units. Therefore, it is critical to consider overall system design to satisfy the increasing demand for nextgeneration cellular systems. To this end, the antenna not only requires high-gain and high-efficiency antenna elements, but also operates effectively as an antenna system together with the baseband signal processing. Thus, this special issue includes papers related to antenna design and system technologies for next-generation cellular systems, where "antenna design" and "antenna system" as well as their joint design are investigated. We would like to thank all the authors for their excellent contributions. We also grateful to acknowledge the reviewers for their careful examination of the papers and for the constructive comments they have provided.

This special issue contains eleven papers, where three papers are related to transmit antenna design and two papers investigate the receiver design. Two papers address the interference mitigation, and two papers study the cooperative network. Finally, two papers cover the channel estimation techniques.

In the paper entitled "Refinement method for weighting scheme of fully spatial beamformer," Ch. Bunsanit et al. present a concept to reduce the range of weighting coefficients; hence the hardware realization becomes practical. In this paper, a full prototype of wideband spatial beamformer is constructed to reflect the true beamforming performance of the proposed refinement method. Its radiation patterns obtained from simulation and measurement are compared. As a result, the attenuation or amplification range is reduced, while some radiation characteristic is remained.

In the paper entitled "Superimposed training-based channel estimation for MIMO relay networks," X. Xu et al. apply the superimposed training strategy into the multiple-input multiple-output (MIMO) amplify-and-forward (AF) oneway relay network (OWRN) to perform the individual channel estimation at the destination. Through superposition of a group of training vectors at the relay under power allocation, estimates of the source-relay and relay-destination channels can be obtained directly at the destination. The closed-form Bayesian Cramér-Rao lower bound (CRLB) is derived and used to guide the design of optimal training vectors. The 
simulation results have been provided to verify the Bayesian CRLB results by the normalized mean-square error (NMSE) performance of a suboptimal channel estimator to verify the Bayesian CRLB result.

In the paper entitled "Efficient capacity-based joint quantized precoding and transmit antenna selection using crossentropy method for multiuser MIMO systems," J.-C. Chen et al. present a cross-entropy-based scheme that realizes the joint precoding and transmit antenna selection in the downlink of MU-MIMO systems with limited feedback to reduce the interference effectively and lower the required RF chains. With the aid of the cross-entropy method, the large amount of search required can be successfully reduced. The simulations demonstrate that the proposed cross-entropy method not only provides better capacity performance but also enjoys complexity advantages compared with the conventional genetic algorithm method.

In the paper entitled "Joint frequency and 2D-DOA estimation for L-shaped array using iterative least squares method," L.-Y. Xu et al. propose an iterative least squares method for estimating the 2D-DOA and frequency based on L-shaped array. Without spectral peak searching and pairing, this algorithm works well. In addition, the proposed algorithm has much better 2D-DOA and frequency estimation performance than conventional ESPRIT algorithm and propagator method, and it has a very close $2 \mathrm{D}-\mathrm{DOA}$ and frequency estimation performance to MUSIC algorithm.

In the paper entitled "Partial PIC-MRC receiver design for single carrier block transmission system over multipath fading channels," J.-H. Deng and Sh.-Y. Huang present a low complexity partial parallel interference cancellation (PIC) with maximum ratio combining (MRC) technology for receiver design to combat the intercarrier interference (ICI) problem over multipath fading channel. With the aid of MRC scheme, the proposed partial PIC technique can effectively perform the interference cancellation and acquire the benefit of frequency diversity gain. Finally, the proposed system can be extended to use for the single-input multiple-output (SIMO) system to provide excellent performance. Simulation results reveal that the proposed low complexity partial PICMRC SIMO system can provide robust performance and outperform the conventional PIC and the iterative frequency domain decision feedback equalizer (FD-DFE) systems over multipath fading channel environment.

In the paper entitled "Layered capacity-based relay-andantenna joint selection for MIMO AF multiple-relay systems in correlated channels," C.-C. Hu et al. present computationally efficient two-stage greedy capacity maximization (GCM) relay-and-antenna joint selection for a dual-hop AF-MIMO multiple-relay system over correlated fading channels. From simulation results, it is obvious that a considerable reduction in system hardware cost and signal processing complexity is achieved by the proposed scheme. In addition, the modified GCM method provides similar capacity performance to the GCM scheme and achieves better capacity performance than those of the conventional techniques.

In the paper entitled "Antenna array design for LOSMIMO and gigabit ethernet switch-based Gbps radio system," $\mathrm{Ch}$. Zhou et al. address issues in antenna array design in the line-of-sight (LOS) environments, where antennas are usually highly correlated and hence MIMO capacity gain cannot be achieved. With the objective of achieving full multiplexing gain, an antenna array design rule is analyzed and proposed to guarantee full multiplexing gain for LOS-MIMO systems, for both one-dimensional and two-dimensional antenna arrays. The proposed design rule relaxes the strict perpendicular constraint in the two-dimensional case. Based on the proposed design rule, the minimum antenna array area and the performance sensitivity to the area error are obtained for practical system design. A demo MIMO-OFDM system at $15 \mathrm{GHz}$ carrier frequency is then implemented demonstrating higher than $15 \mathrm{bps} / \mathrm{Hz}$ spectrum efficiency.

In the paper titled "Improved sparse channel estimation for cooperative communication systems," G. Gui et al. consider the amplify-and-forward (AF) cooperative communication system (CCS), which is implemented by two time slots: in the first slot, signal is broadcasted from source $S$ to relay $R$ and destination $\mathrm{D}$, and in the second slot, only the amplified signal is forwarded $D$. Both cascaded channel ( $\Rightarrow R \Rightarrow$ D) and direct channel $(S \Rightarrow D)$ are acquired at the $D$. The cooperative channel consists of two parts: sparse channel $(S \Rightarrow D)$ and dense one $(S \Rightarrow R \Rightarrow D)$. Based on the partial sparse assumption of the cooperative channel model, an improved channel estimation method is proposed with partial sparse constraint. By using sparse decomposition theory, channel estimation is first formulated as a compressive sensing problem. The cooperative channel is then reconstructed by least absolute shrinkage and selection operator (LASSO) with partial sparse constraint. Numerical simulation results confirm the superiority of proposed methods over global sparse channel estimation methods.

In the paper entitled "Interference mitigation framework for cellular mobile radio networks," W. Mennerich et al. present a framework for interference mitigation in cellular mobile radio networks. Based on analysis of interference conditions, scheduling effects, and so forth, different concepts are considered, for example, joint transmission (JT) coordinated multipoint (CoMP), interference rejection combining (IRC), multiuser MIMO (MU-MIMO), interference floor shaping, wideband beamforming, antenna tilting, power adaptation, clustering, user grouping, effective precoding, per cell and per cooperation area scheduling, and so on. Simulation results show 2.4-times higher spectral efficiency by the proposed framework over MU-MIMO and 1.8 times higher than JTCoMP. The simulation results are also verified with those from the test bed based in Technical University of Dresden, confirming the viability of the proposed framework in real systems.

In the paper entitled "A multistage decision-feedback receiver design for LTE uplink in mobile time-variant environments," J.-H. Deng and Sh.-M. propose a multistage decisionfeedback structure to mitigate the ICI effect and enhance system performance in time-variant environments. Based on the block-type pilot arrangement of the LTE uplink type 1 frame structure, the time domain least square (TDLS) method and polynomial-based curve-fitting algorithm are employed for channel estimation. Instead of using a conventional equalizer, this work uses a group frequency domain equalizer (GFDE) 
to reduce computational complexity. Furthermore, this work utilizes a dual iterative structure of group parallel interference cancellation (GPIC) and frequency-domain group parallel interference cancellation (FPIC) to mitigate the ICI effect. Finally, to optimize system performance, this work applies a novel error-correction scheme. Simulation results demonstrate that the bit error rate (BER) performance is markedly superior to that of the conventional full-size receiver based on minimum mean square error (MMSE). This structure performs well and is a flexible choice in mobile environments using the SC-FDMA scheme.

In the paper entitled "Interference-based decode and forward scheme using relay nodes in heterogeneous networks," K. Nishimori et al. present a scheme that aims to reduce the interference from the pico BS to the macro UE in the LTE setup. The system is designed to use a relay station to help detect the interference signal from the pico BS first and then forwards it to the macro UE to conduct interference cancellation. This can obtain better performance for the signal detection at the macro UE.

Chih-Peng Li Sumei Sun Ding-Bing Lin Jung-Chieh Chen Qiang Chen 

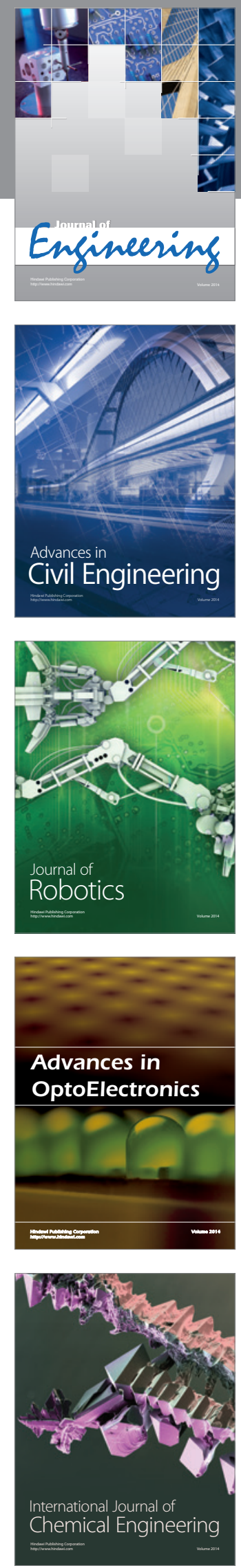

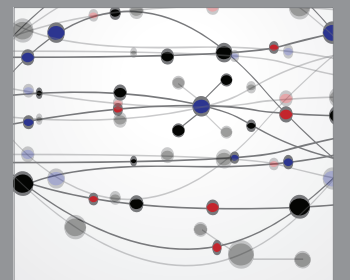

The Scientific World Journal
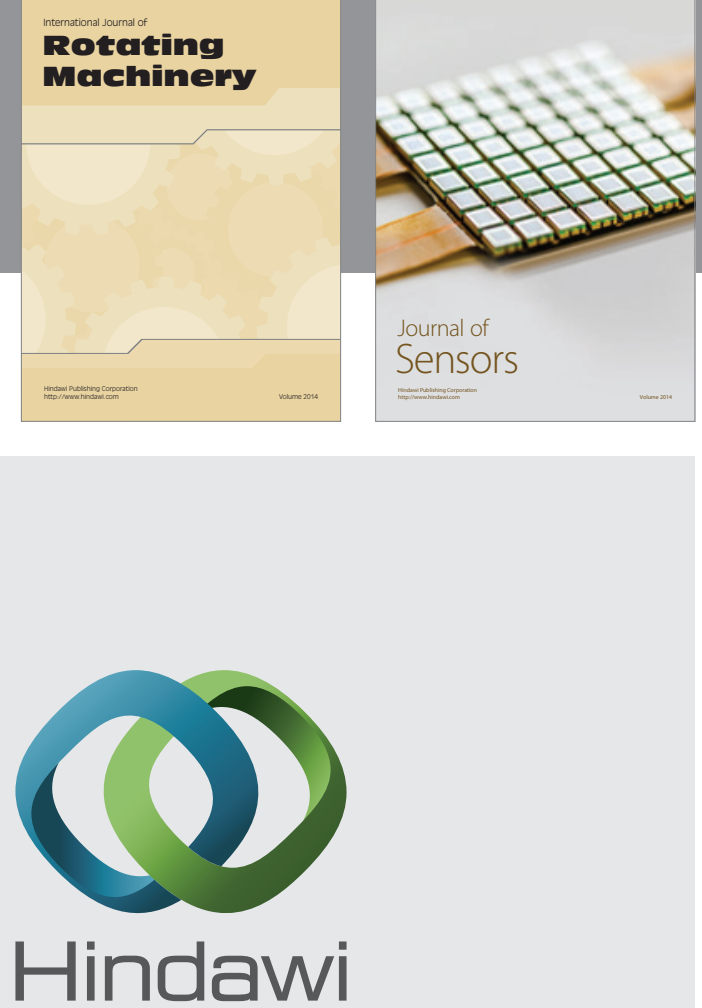

Submit your manuscripts at http://www.hindawi.com
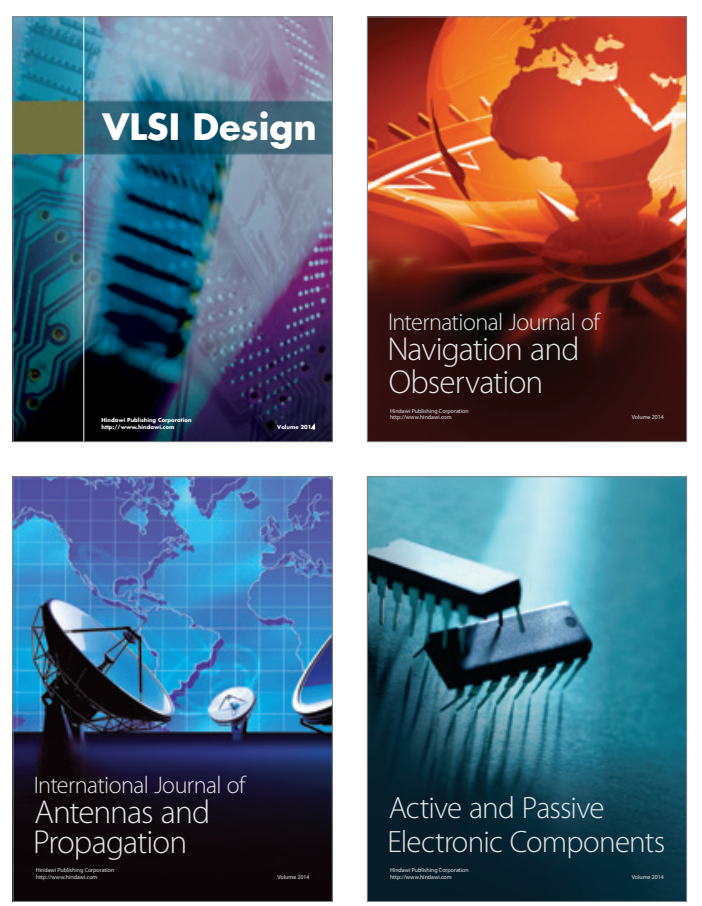
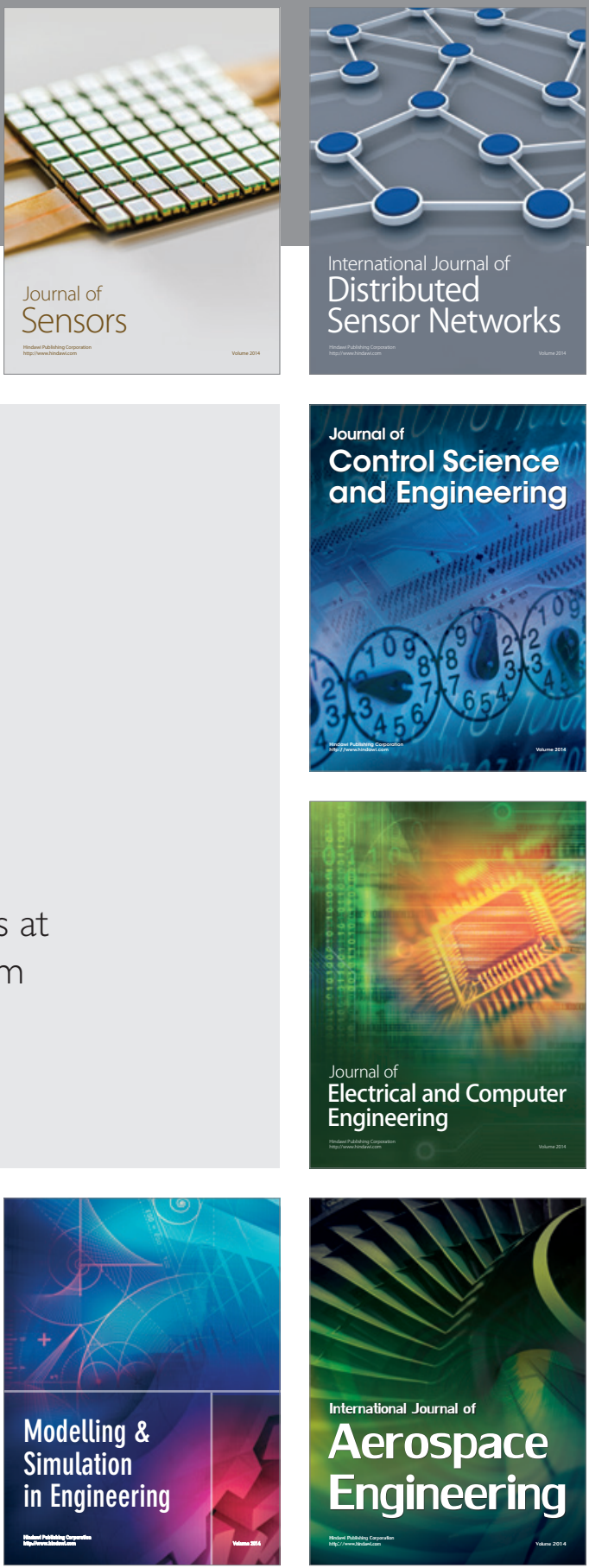

Journal of

Control Science

and Engineering
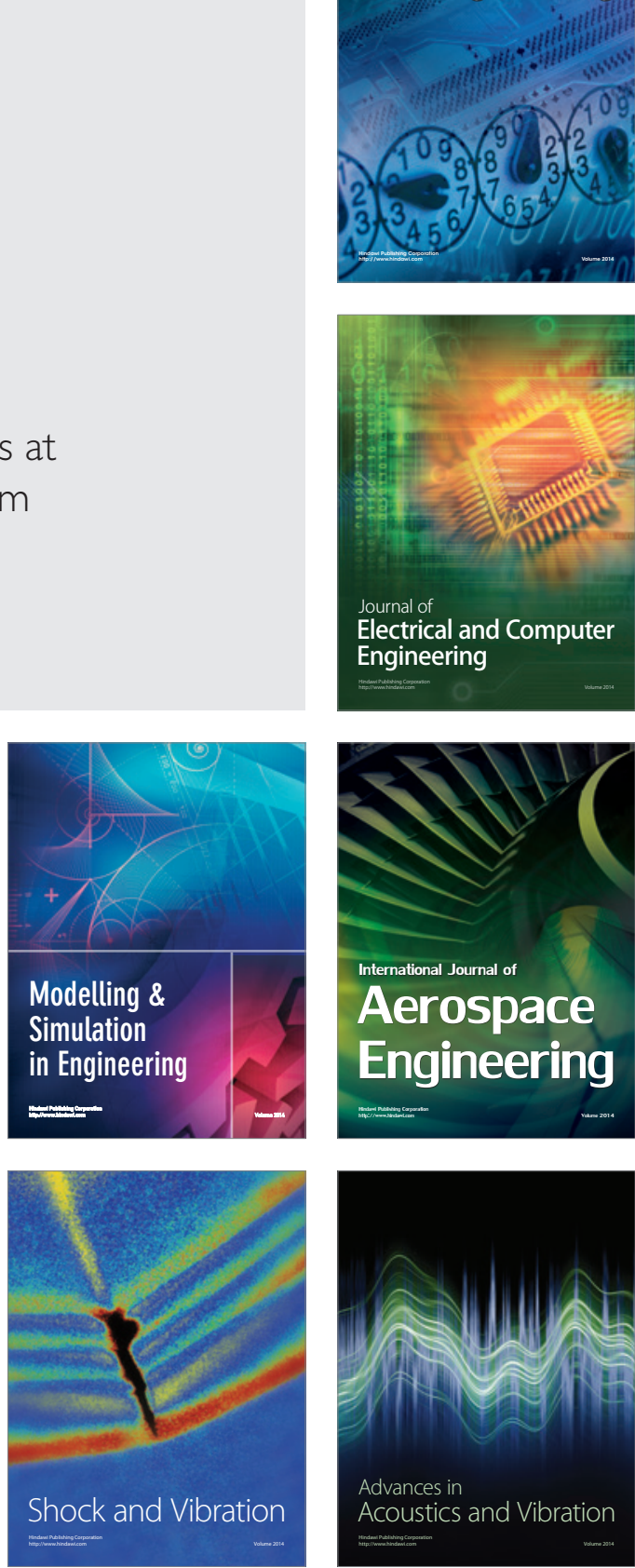